\title{
Effects of the reproduction number in a SEIIRD model describing the time evolution of COVID-19 at country level
}

\author{
FLAVIUS GUIAS, \\ Dept. of Mechanical Engineering \\ Dortmund University of Applied Sciences and Arts \\ Dortmund, GERMANY
}

\begin{abstract}
We consider a compartmental model of SEIIRDtype which describes the time evolution of the COVID-19 epidemy at the level of a country. For the reproduction number $\mathrm{R}(\mathrm{t})$, the crucial parameter which influences the number of new cases, we consider an explicit form as a combination of trigonometric, exponential and gaussian functions. The coefficients of the individual parts can be adapted in order that the profile of $\mathrm{R}(\mathrm{t})$ matches different scenarios. Their common structure illustrates the real behaviour observed in most countries. Initially we can observe large values of $\mathrm{R}(\mathrm{t})$ which enforce the first wave of the epidemy, followed by a rapid reduction below 1 due to a first lockdown which can have different intensities. The second phase consists of a relaxation of the restrictions having as a consequence an increase of the reproduction number within a range over 1 . The numerical simulations show that in this case, after a period of some months with a low level of daily cases, the occurrence of a second wave is unavoidable, being inherent to the nature of the model. The intensity of the second wave depends on how much and how long the reproduction number $\mathrm{R}(\mathrm{t})$ has been over the threshold value of 1 , but also on the intensity of the first lockdown. All simulations show that the behaviour of the model is very sensitive with respect to the reproduction number. Small changes in its values may have a significant impact on the long-term evolution of the epidemy at the country-level.
\end{abstract}

Index Terms - compartmental model in epidemiology, ordinary differential equations, SEIIRD, COVID-19

Received: September 14, 2020. Revised: December 20, 2020. Accepted: December 27, 2020. Published: December 31, 2020.

\section{Introduction}

The basic approach used for the modeling of epidemies is the SIR-model, see [1], [2]. It belongs to the so-called compartmental models, since the population is divided into several compartments: S - susceptible, I - infected and R - recovered. The dynamics occurs in several stages, by transitions from one stage to the next. Susceptible individuals can get the disease by contact with infected ones. The corresponding rate is directly proportional to the numbers $S$ and $I$, while the transition from $I$ to $R$ occurs at a rate proportional to the number $I$ of infected individuals. A person in this last stage is already immune and cannot contribute anymore to the spread of the disease. The system of ordinary equations which corresponds to the basic SIR model is the following:

$$
\begin{aligned}
\frac{d S}{d t} & =-\beta \cdot I \cdot \frac{S}{N} \\
\frac{d I}{d t} & =\beta \cdot I \cdot \frac{S}{N}-\alpha \cdot I \\
\frac{d R}{d t} & =\alpha \cdot I
\end{aligned}
$$

The number $N$ denotes the size of the whole population and the presence of the factor $N^{-1}$ is necessary for the correct scaling of the bilinear terms. The coefficients of the linear terms, i.e. the rates of passing from one state to another, are inversely proportional to the average time spent in the corresponding state. With $T_{\text {inf }}$ being the average time spent in the infectious state, we can therefore assume that $\alpha=T_{\text {inf }}^{-1}$, while for the transmission rate $\beta$ we can consider the form $\beta=R(t) \cdot T_{\text {inf }}^{-1}$. The term $R(t)$ denotes the time dependent effective reproduction number, i.e. the average number of further infections produced by the contacts with one infectious individual. At the beginning of the epidemy its value is equal to $R_{0}$, the so called basic reproduction number, but after that it may vary due to restrictions, social distancing, increased frequency of testing, etc.

The equilibria of system (1) are given by the property $I=0$, while $S$ and $R$ are constants with $S+R=N$. The second equation can be written as

$$
\frac{d I}{d t}=\left(R(t) \cdot \frac{S}{N}-1\right) \cdot I \cdot T_{\text {inf }}^{-1},
$$

therefore, if for all $t$ we have $R(t) \cdot S / N-1<0$, in particular if $R(t)<1$, then we have $d I / d t<0$, so the epidemy will vanish in a short time, since the number of initially infected individuals decays exponentially. However, if at a certain time $t$ we have $R(t) \cdot S / N-1>0$, then the epidemy will eventually spread among a large part of the population, since the size of the initially infected population grows exponentially starting with the beginning of the dynamics, till either $S(t)$ or $R(t)$ will become small enough in order to ensure that $d I / d t<0$.

In the long-time behaviour, the solutions will approach in both cases an equilibrium state with $I=0$ and constant values of $S$ and $R$, which depend on the initial condition 
and on the time-dependent reproduction number $R(t)$. The stability analysis of the SIR model is performed in paper [3] along that for related systems, with additional transitions corresponding to birth, death or reinfection. In turns out that in the enhanced models, besides the trivial equilibria with $I=0$ (stable if $R(t) S / N<1$, in particular if $R(t)<1$ for all $t$, and unstable if $R(t) S / N>1$ ), there exists also a nontrivial endemic equilibrium state whose stability is shown by a Lyapunov function.

The SEIR model, see [1], [2], considers the additional state $E$ of exposed individuals during the incubation time, situated between $S$ and $I$, where they don't show yet the symptoms and cannot spread the disease.

In context of the outbreak of the COVID-19 pandemy at the beginning of 2020, several compartmental models of type SIR, SEIR and modifications of them were applied in order to describe the evolution of this disease. The papers [4] and [5] present an overview of such models with applications to COVID-19. Applications to concrete countries are presented in [6], [7], [8], [9], where the authors employ extensions of the SEIR model. In the latter reference, the parameters of the model were estimated using a particle swarm optimization (PSO) algorithm.

The paper [10] considers a SIIRD model with timedependent transmission rate in order to account for the features of the COVID-19 disease and for the publicly available statistical data, which are used to fit the parameters of the model. The statistics record the numbers of new infections, the current infections, the recoveries and the deaths. Since data for $E$ are not available, a model of SEIR type was not considered. Instead, a component $D$ was added, which counts the death cases and transitions into $D$ were considered from the state $I$ and sometimes directly from $S$, in the case of infections reported only after the death of the individual. In the considered model, the infectious state is split into two stages. In the first one, $I_{1}$, which lasts relatively short, the individual can spread the disease, while death is unlikely to occur. The following stage $I_{2}$ lasts until the individual is reported as recovered or dead and for this category it is assumed that it does not contribute to the spread of the disease. The parameter estimation method used in the mentioned paper employs a numerical solver for the ODE system which uses as predictor the path of a stochastic jump process which is based on the variations of the data set, after which the precision is improved by using correction steps of RungeKutta type. The solutions computed in this way are subjected to an optimization procedure which searches for the set of parameters which minimizes the mean square error between the computed and the given data.

In the present paper we consider a SEIIRD model as an extension of the model in [10]. That is, we add also the state of exposed individuals, accounting for the incubation period. The corresponding system of ordinary differential equations together with the considered values of the parameters is presented in Section II. The goal is to perform several numerical simulations in order to analyze the influence of the reproduction number $R(t)$, that is of the average number of new infections produced by one individual, on the evolution of the epidemy. For this purpose we consider the following form:

$$
\begin{aligned}
R(t)= & c_{0} \cdot\left(e^{-0.01 t^{2}}+e^{-0.001 t}(\cos (0.05 t)+0.1 \sin (0.5 t))\right. \\
& +0.025+c_{60} e^{-0.0001(t-60)^{2}} \\
& +c_{120} e^{-0.001(t-120)^{2}}+c_{190} e^{-0.001(t-190)^{2}} \\
& +c_{250} e^{-0.001(t-250)^{2}}+c_{300} e^{-0.001(t-300)^{2}} \\
& \left.+c_{340} e^{-0.001(t-340)^{2}}\right)
\end{aligned}
$$

$c_{0}$ is a scaling constant and the first row of the expression above describes the basic shape of $R(t)$. The second row ensures that its values remain positive and the coefficient $c_{60}$ controls the magnitude of the first lockdown, while the coefficients of the gaussian functions in rows 3-5 can be properly adjusted in order to simulate several scenarios at later stages of the epidemy. Typical profiles can be seen in Figure 2. The goal is to describe the behaviour which could be observed in several countries. At the beginning of the epidemy, the values of the reproduction number were well over 1 , but due to timely restrictions followed by a lockdown, $R(t)$ fell under the threshold value of 1 . In the next phase of gradual relaxations, its values increased again slightly over 1 , remaining in this range for a period of several months. After this time span with relatively low infection numbers, a second wave of the epidemy emerged, in most cases significantly larger than the first one.

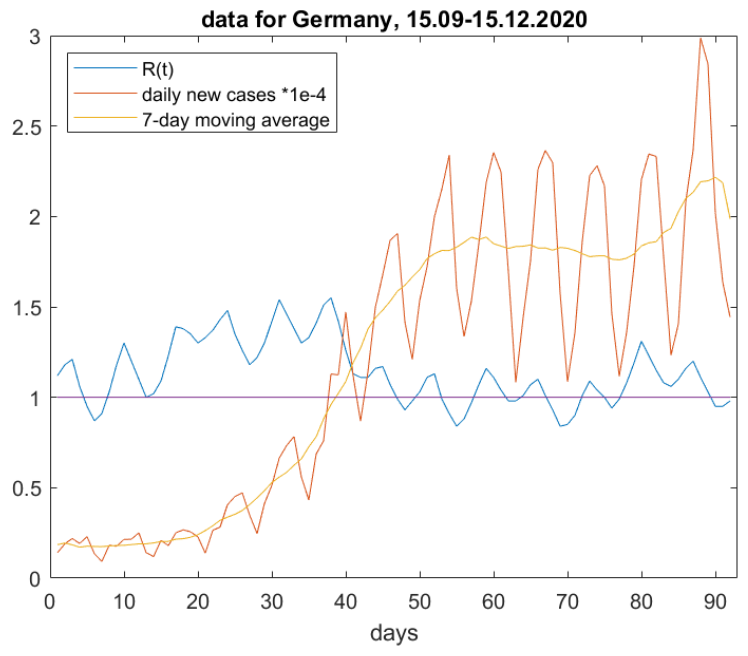

Fig. 1. Evolution in Germany between 15.09-15.12.2020

This behaviour at later stages for Germany is illustrated in Figure 1. After the lockdown during the first wave in MarchApril 2020, the number of new infections remained for several months at a relatively low level, although the reproduction number $R(t)$ was constantly larger than 1 . Starting with the middle of October, the second wave began to emerge, which led to a partial lockdown between 1.11-15.12.2020. The effects 
on the reproduction number can be seen also in the picture, where starting with the 45th day of the considered time span the values of $R(t)$ were pushed significantly downwards, but oscillating around the treshold value of 1 . This is reflected in relatively constant figures of daily new infections till the beginning of December, when the reproduction number jumped again at a level significantly over 1 . The consequence was a further increase of the daily new infections, which motivated the introduction of a harder lockdown starting with 16.12.2020, but whose effects aren't captured by this picture.

In Section III we present the results of the numerical simulations of the SEIIRD model in the case of three scenarios with successive reduction of the reproduction number $R(t)$ in order to analyze the impact on the occurrence and on the magnitude of the second wave of the epidemy. The basic profiles are illustrated in Figure 2. The differences between them consist in the magnitude of $R(t)$ on the interval between ca. 200-350 days. We start with a profile $R(t)>1$ in this interval, a property which holds also for the second one, but with smaller values, while the third profile is the smallest, being even $<1$ for $t>280$.

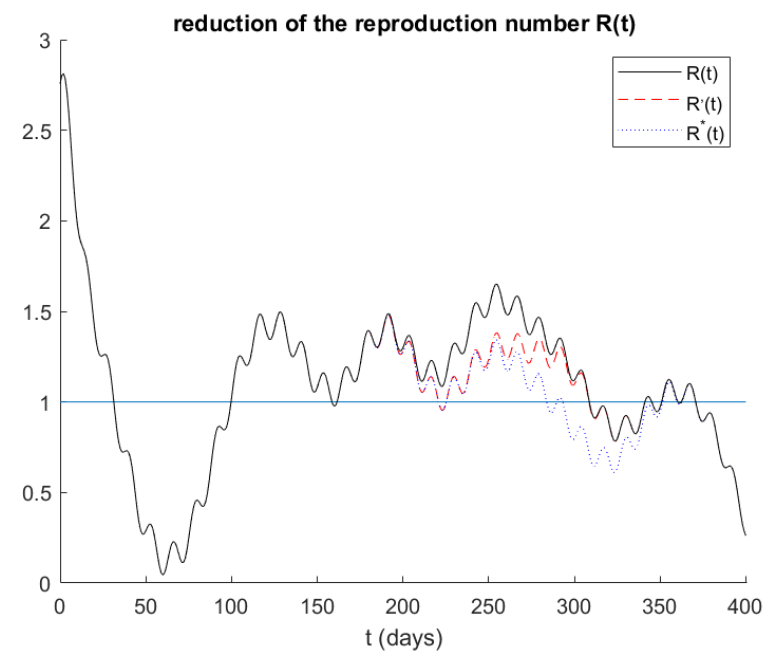

Fig. 2. The standard profiles of $R(t)$ in each of the three scenarios

These properties are obtained by modifying $R(t)$, the standard profile used in the first scenario. For $R^{\prime}(t)$ we multiply $c_{250}$ with the factor 0.5 in order to simulate the second scenario of additional mild restrictions. For $R^{*}(t)$ we additionally multiply $c_{300}$ with the factor 0.8 in order to push the reproduction number below 1 .

In each of these three scenarios we consider three variants of the profile of $R(t)$ (the exact values of the parameters will be given in the corresponding subsections). The standard profile in each scenario has $c_{0}=1$ and corresponds to those presented above and depicted in Figure 2. The second profile in each scenario has the same parameter values except $c_{0}=1.02$, that is, we consider values of $R(t)$ which are only with $2 \%$ larger. The simulations show that the results are extremely sensitive with respect to the reproduction number and that a small modification, as in this example, leads to significant changes in the behaviour. More precisely, in the latter case, the amplitude of the second wave will turn out to be considerably larger. The third profile corresponds to proper adjustments of the parameters, such that the magnitude of the first lockdown (which can be seen in Figure 2 around $t=60$ days) is smaller, that is, the profile of $R(t)$ is here above the standard one, but at later times always slightly below it. It will turn out that a milder lockdown at the beginning of the spread of the epidemy has an important influence on the second wave, which appears earlier and has a larger magnitude than in the case of the standard profile.

As a general conclusion of the simulations we can state that the profile of the reproduction number $R(t)$ has a significant influence on the evolution of the epidemy, the model being highly sensitive regarding this parameter. After the first lockdown, when the values of $R(t)$ climb again beyond 1 , the apparition of the second wave is inevitable, although the daily new infections might stay at low levels for several months. The moment of emergence and the magnitude of the second wave depend on how much and how long the reproduction number $R(t)$ has been over the threshold value of 1 , but also on the intensity of the first lockdown.

\section{The Seiird Model}

In this paper we consider the following system of ordinary differential equations:

$$
\begin{aligned}
\frac{d S}{d t} & =-\frac{R(t)}{T_{i n f}} \cdot I_{1} \cdot \frac{S}{N}-\tilde{\mu}_{d} \cdot S \\
\frac{d E}{d t} & =\frac{R(t)}{T_{i n f}} \cdot I_{1} \cdot \frac{S}{N}-T_{i n c}^{-1} \cdot E \\
\frac{d I_{1}}{d t} & =T_{i n c}^{-1} \cdot E-T_{i n f}^{-1} \cdot I_{1} \\
\frac{d I_{2}}{d t} & =T_{i n f}^{-1} \cdot I_{1}-T_{c o n v}^{-1} \cdot I_{2}-\mu_{d} \cdot I_{2} \\
\frac{d R}{d t} & =T_{\text {conv }}^{-1} \cdot I_{2} \\
\frac{d D}{d t} & =\mu_{d} \cdot I_{2}+\tilde{\mu}_{d} \cdot S
\end{aligned}
$$

These equations correspond to the following states: $S$ susceptible, $E$ - exposed (infected individuals during the incubation time), $I_{1}$ - individuals during the infectious state, when they can spread the disease, $I_{2}$ - individuals which are still counted as "active cases", but which are not infectious anymore, $R$ - recovered and $D$ - dead.

$R(t)$ is the time-dependent reproduction number, $T_{i n c}$ the average incubation time, $T_{i n f}$ the average time spent in the infectious state and $T_{\text {conv }}$ the average convalescence time, spent in the state $I_{2}$. Deaths are considered to take place either directly from the state $S$ at rate $\tilde{\mu}_{d}$ or from the state $I_{2}$ at rate $\mu_{d}$.

The precise form of $R(t)$ will be given in the next section. For the average incubation time we take $T_{i n c}=5$, while for the other parameters we consider the following values, 
which are of the same order as those computed in [10] based on the statistical data for several countries: $T_{\text {inf }}=$ $4, T_{\text {conv }}=14, \mu_{d}=5 \cdot 10^{-3}, \tilde{\mu}_{d}=10^{-6}$. The population is considered to be $N=4 \cdot 10^{7}$ individuals and we consider as initial conditions: $S(0)=N-900, E(0)=600, I_{1}(0)=$ $300, I_{2}(0)=R(0)=D(0)=0$.

Our goal is to perform numerical simulations in several scenarios involving different profiles of $R(t)$ in order to obtain a qualitative comparison of the dynamics of the spread of the infection among the population of a generic country. The most important parameter which accounts for this and which is also presented in all statistics is the number of daily new infections. Therefore, the focus of the diagrams of the next section will be put on this number, given in our model by $n_{t}=\int_{t}^{t+1} T_{i n c}^{-1} \cdot E(s) d s$ and which is computed by numerical integration of the approximation of the component $E$ of the system (3). Its numerical solution as well as the integrals are computed using MATLAB. The settings for the solver ode 45 were set in order to obtain a high precision, i.e. very small, adapted, time discretization steps and the integrals were computed using the trapezoidal rule over a nonuniform grid.

\section{Numerical Simulations}

In this section we will perform numerical simulations for three different scenarios corresponding to different degrees of reduction of the reproduction number $R(t)$ after $t=200$ days, in order to analyze the impact on the possible second wave. In each particular scenario we compare the results for three different profiles of the reproduction number $R(t)$ : a standard one, a profile scaled with the factor 1.02 and a third profile which initially (around $t=60$ days) has a less significant reduction of $R(t)$, which corresponds for example to a less severe lockdown, but which afterwards has similar values or even slightly smaller than the standard profile.

The general form of $R(t)$ is given by (2) and the exact values of the parameters are given in each of the next subsections.

\subsection{Scenario 1}

For the basic profile of the reproduction number $R_{1}(t)$ we consider $c_{0}=1, c_{60}=1.05, c_{120}=-0.2, c_{190}=$ $2, c_{250}=0.55, c_{300}=1.5, c_{340}=0.8$. The second simulation is performed for $1.02 \cdot R_{1}(t)$, i.e. for $c_{0}=1.02$ while all other parameters are the same as before and for the third simulation $R_{2}(t)$ corresponds to the values $c_{0}=0.9, c_{60}=1.3, c_{120}=$ $-0.2, c_{190}=2, c_{250}=0.55, c_{300}=1.5, c_{340}=0.9$. This latter choice ensures that the initial reduction around $t=60$ days is not as significant as for the basic profile, but at later times this modified profile does not exceed the former one.

In this first scenario all three considered profiles for the reproduction number are in a time interval approximately between days 100 and 300 continuously over the critical threshold of 1, see Figure 3.

The results of the simulations are depicted in Figure 4.

We can note that, due to the fact that the reproduction number stays constantly over 1 for ca. 200 days, the emergence

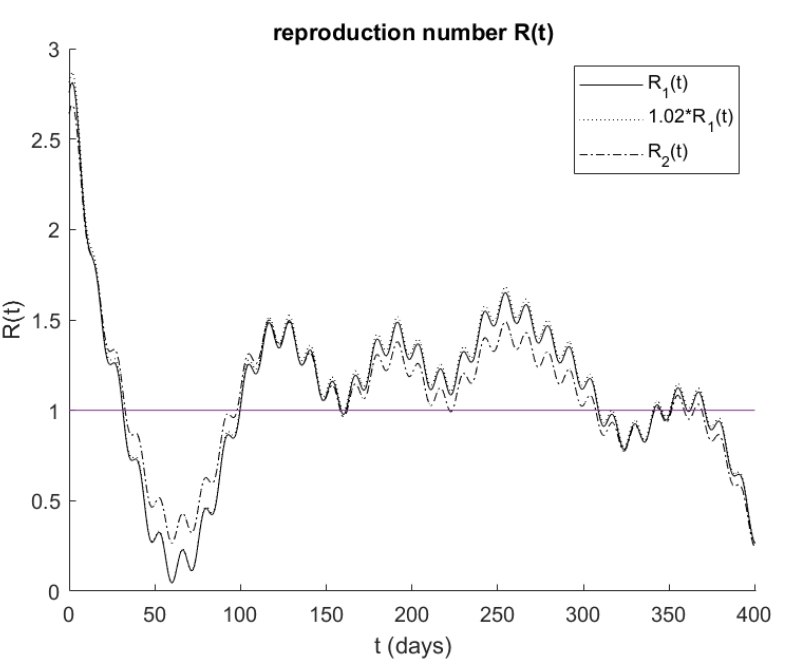

Fig. 3. The profiles of $R(t)$ in Scenario 1

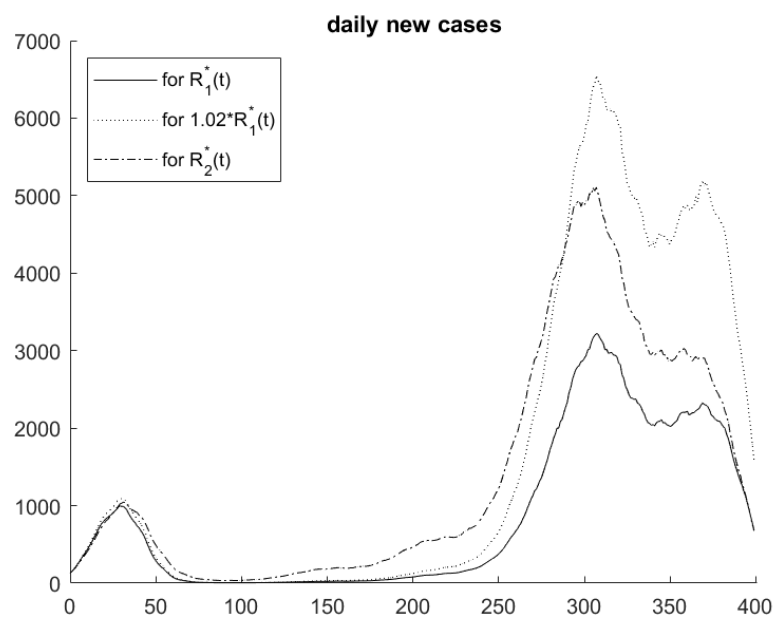

Fig. 4. Daily new cases in Scenario 1

of a second wave of new infections, significantly larger than the first one, is inevitable. This is the case although the number of daily new cases is for a relatively long time, between days 100-250, significantly lower as in the first wave, especially for $R_{1}(t)$ and $1.02 \cdot R_{1}(t)$. Comparing the magnitude of the second wave, we remark that the dynamics of the infection is very sensitive with respect to the reproduction number. By scaling the profile with the factor 1.02, the second wave is about two times larger than in the case of the original profile of $R_{1}(t)$. Moreover, the second wave corresponding to $R_{2}(t)$ occurs earlier and is significantly larger than the reference one, although the first wave is quite similar. The reason for this behaviour lies in the fact that, due to the less severe lockdown around $t=60$, at the end of the first wave we have more infectious individuals than in the standard case.

The conclusions of the simulations in the frame of this first scenario can be summarized as follows. The model exhibits 
a high degree of sensitivity with respect to the reproduction number $R(t)$. If its values stay long enough over the critical threshold of 1 , the occurrence of a second wave is inherent to the model, even if it can stay far from such a behaviour for several months. This phenomenon could be observed in many countries, a particular case being Germany, as shown in Figure 1. The magnitude of the second wave depends however strongly on the particular form of $R(t)$, even if in the case of the first wave the differences were almost negligible.

A substantial reduction below 1 of the reproduction number during the early stages of the epidemy can delay the occurrence of the second wave and significantly lower its magnitude. This can be a possible explanation for the situation in China, where in the first months a severe lockdown was imposed, but after some time all restrictions could be lifted. The possible new cases which could have appeared in the meantime might be at any moment under control, being immediately isolated and prohibited to spread the disease. Nevertheless, this approach is possible only if the disease is not widespread among the population, a state which was achieved by a strong reduction of $R(t)$ for a certain period, even closer to 0 as in our simulations. On the other hand, even in this situation, if new cases may appear unobserved while their number is not significant enough, the emergence of a second wave is still possible, even if it may appear with a significant delay.

\subsection{Scenario 2}

In the second scenario we consider the same coefficients as in the first one, except for taking $c_{250}=0.275$, i.e. the half of the standard value. Furthermore, we consider here also three different profiles for $R(t)$, similar to scenario 1 .

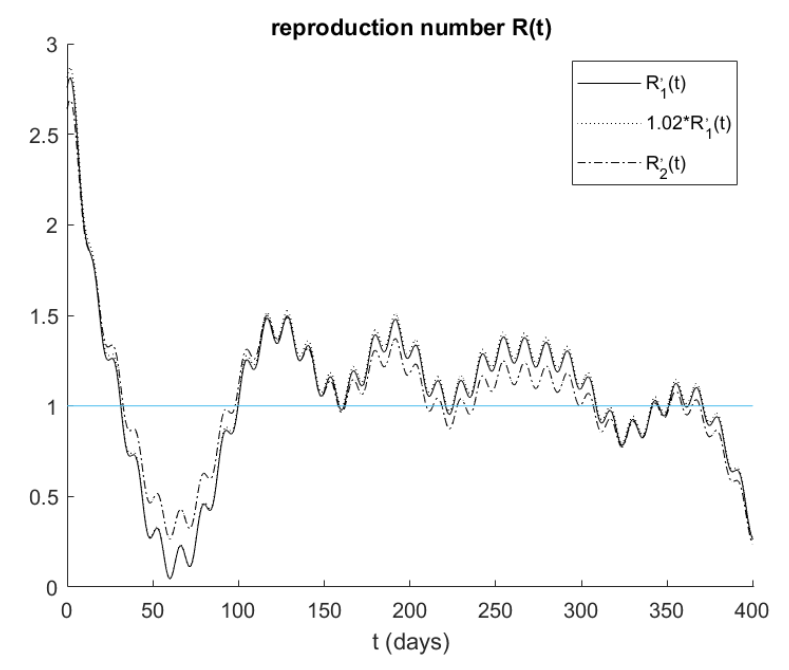

Fig. 5. The profiles of $R(t)$ in Scenario 2

This modification still keeps the profiles of $R(t)$ over 1 during the days 100-300, but the values around $t=250$ are smaller than in the first scenario, as illustrated in Figure 5.

The results of the numerical simulations are plotted in Figure 6. The second wave of infections can be observed also

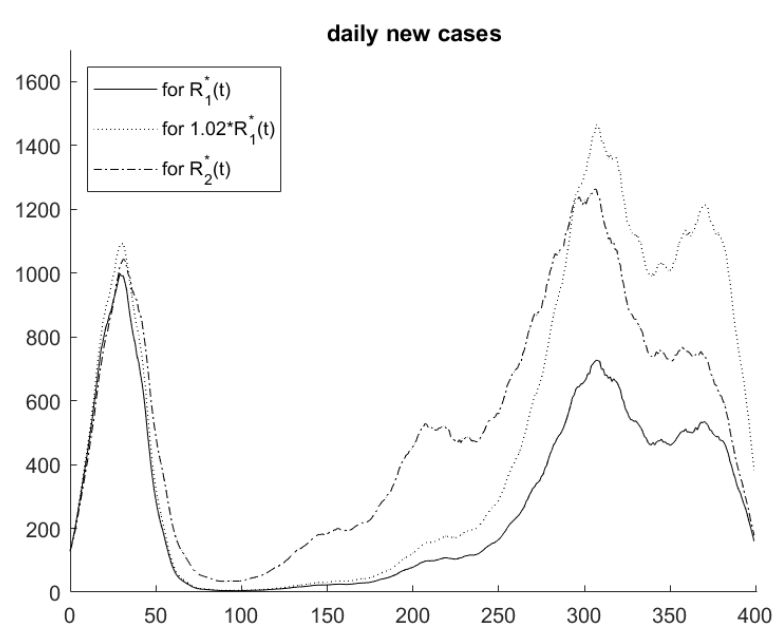

Fig. 6. Daily new cases in Scenario 2

in this case, but the amplitude is much lower than in scenario 1. Its magnitude is comparable to that of the first wave, in the case of the standard profile $R^{\prime}(t)$ being even smaller.

The conclusion is that a proper reduction of the reproduction number $R(t)$ about one month before the emergence of the second wave, even if it still remains above 1 , will lead to a milder second wave of infections compared to the basic scenario.

\subsection{Scenario 3}

In the third scenario we consider the same coefficients as in the basic one, except for taking $c_{250}=0.275$ and $c_{300}=1.2$. That is, in addition to the second scenario, we further lower the profile of $R(t)$, bringing it around $t=300$ below the critical treshold of 1, as can be seen in Figure 7.

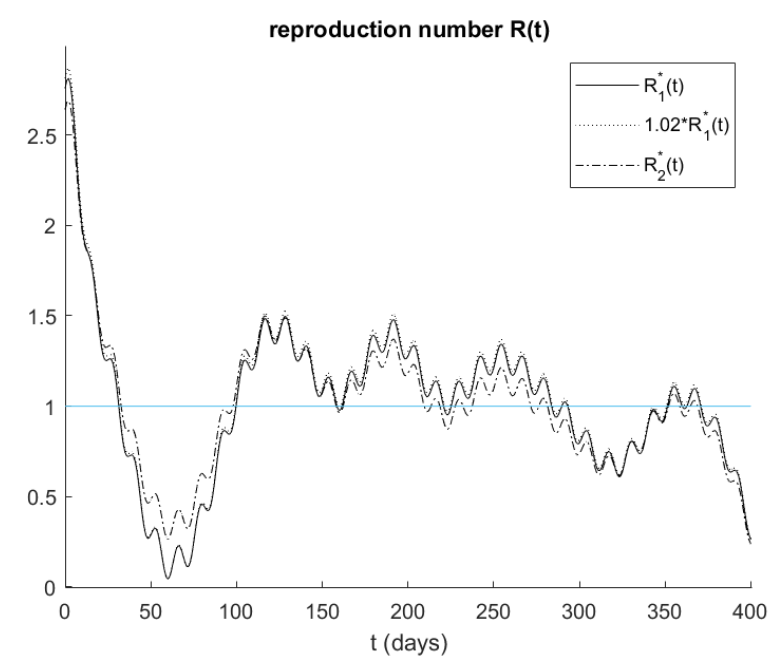

Fig. 7. The profiles of $\mathrm{R}(\mathrm{t})$ in Scenario 3

In this case, as shown in Figure 8, the magnitude of the second wave is for all three profiles of $R(t)$ lower than that 
of the first wave.

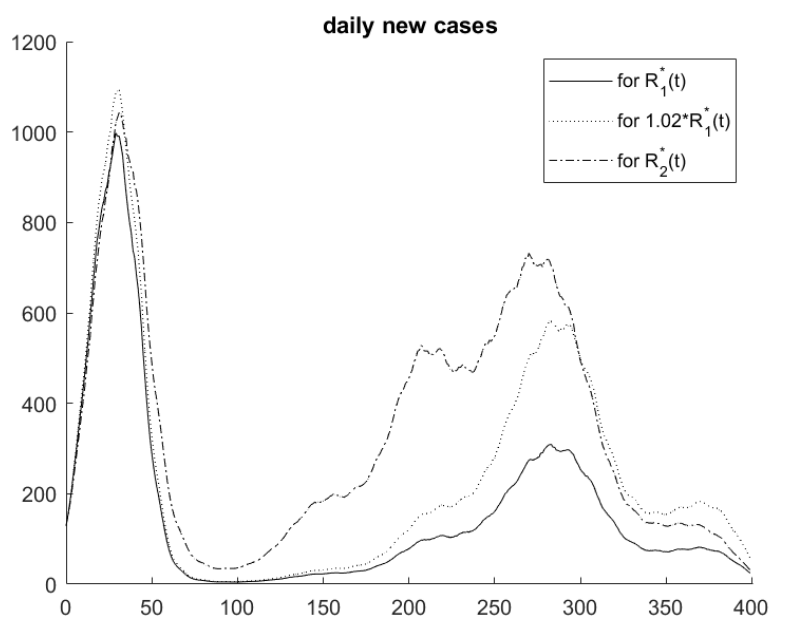

Fig. 8. Daily new cases in Scenario 3

\section{Conclusion and Discussion}

In this paper we considered a SEIIRD system of differential equations modeling the dynamics of the COVID-19 pandemy at the level of a generic country. The goal was to analyze the dependance of the new infection cases on the reproduction number $R(t)$ by performing numerical simulations in different scenarios. For the different profiles of $R(t)$ we considered an explicit form as a combination of trigonometric, exponential and gaussian functions. The coefficients of the individual parts can be adapted in order that the profile of $R(t)$ matches different scenarios.

The results exhibited a high sensitivity with respect to this parameter. Small global or local changes can lead to major differences in the dynamics of new infections at later times. They influence especially the magnitude of the second wave, which emerges if the reproduction number stays for a certain time (a couple of months) above the critical threshold of 1, even if during this period the number of daily new cases might stay at a relatively low level.

Nevertheless, the amplitude of the second wave can be significantly reduced either if the intensity of the lockdown at an early stage is strong enough, within the same duration, or if one manages to reduce the reproduction number $R(t)$ at a right moment before the occurrence of the second wave. Based on the present numerical simulations, we can observe empirically that the second wave emerges after a period in which $R(t)$ was for long enough period above 1 .

A further research topic could be therefore a more rigorous quantification of this fact. Quantities of interest might be $L^{p}$-norms of $R(t)-1$ on time intervals $\left[t_{1}, t_{2}\right]$ where the reproduction number is above 1 and the goal would be to find critical bounds of these norms which, when exceeded, might be an indicator of the imminent approaching of the second wave. Reducing $R(t)$ before this event effectively occurs could be crucial for controlling its magnitude. Moreover, since the numerical simulations showed that the intensity of the early lockdown can have a significant impact on the second wave, one can analyze a similar norm of the control function $1-R(t)$ on time intervals where it is constantly below 1 and find critical values which, in combination with the previous indicator, can give insight into the magnitude of the second wave.

Additionally, if one would be interested in a long-term simulation, then the model should be enhanced in order to include features like immunity induced by vaccination of the susceptible population, but also losing of immunity after a given time period, regardless if it was obtained by passing through the disease or by vaccination. In this case we expect that the long-time behaviour of the system will approach an endemic equilibrium, which contains a nontrivial amount of infected population.

\section{References}

[1] F. Brauer and C. Castillo-Chávez, Mathematical Models in Population Biology and Epidemiology. Springer, New-York, 2001.

[2] F. Brauer, Compartmental Models in Epidemiology, in F. Brauer, P. van den Driessche and J. Wu (Eds.) Mathematical Epidemiology, Springer, Berlin, Heidelberg 2008, Chapter 2, pp. 19-79

[3] A. Korobeinikov, G.C. Wake, Lyapunov Functions and Global Stability for SIR, SIRS, and SIS Epidemiological Models, Appl.Math.Letters, Vol. 15, 2002, pp. 955-960.

[4] A. Abou-Ismail, Compartmental Models of the COVID-19 Pandemic for Physicians and Physician-Scientists, SN Comprehensive Clinical Medicine, Vol. 2, 2020, pp. 852-858. https://doi.org/10.1007/s42399020-00330-z

[5] A. Anirudh, Mathematical modeling and the transmission dynamics in predicting the Covid-19 - What next in combating the pandemic, Infectious Disease Modelling, Vol. 5, 2020, pp. 366-374. https://doi.org/10.1016/j.idm.2020.06.002

[6] J.M. Carcione, J.E. Santos, C. Bagaini, J. Ba, A Simulation of a COVID-19 Epidemic Based on a Deterministic SEIR Model. Frontiers in Public Health, Vol. 8, 2020, Article 230. https://doi.org/10.3389/fpubh.2020.00230

[7] G. Giordano, F. Blanchini, R. Bruno et al., Modelling the COVID-19 epidemic and implementation of population-wide interventions in Italy. Nature Medicine Vol. 26, 2020, pp. 855-860. https://doi.org/10.1038/s41591-020-0883-7

[8] P. Teles, A time-dependent SEIR model to analyse the evolution of the SARS-covid-2 epidemic outbreak in Portugal, Bulletin of the World Health Organization, E-pub: April 7, 2020. http://dx.doi.org/10.2471/BLT.20.258392

[9] S. He, Y. Peng, K. Sun, SEIR modeling of the COVID-19 and its dynamics. Nonlinear Dyn. Vol. 101, 2020, pp. 1667-1680. https://doi.org/10.1007/s11071-020-05743-y

[10] F. Guiaş, Numerical Simulations of a Modified SIR Model Fitting Statistical Data for COVID-19. WSEAS Transactions in Computer Research, Vol. 8, 2020, pp. 115-125. https://doi.org/10.37394/232018.2020.8.15

\section{Creative Commons Attribution License 4.0 (Attribution 4.0 International, CC BY 4.0)}

This article is published under the terms of the Creative Commons Attribution License 4.0 https://creativecommons.org/licenses/by/4.0/deed.en_US 